\title{
UN MODELO DE FORMACIÓN BASADO EN LAS COMPETENCIAS: HACIA UN NUEVO PARADIGMA EN LA ENSEÑANZA UNIVERSITARIA
}

\author{
Rosa Ma Rodríguez Izquierdo \\ Universidad Pablo de Olavide (Sevilla)
}

\begin{abstract}
RESUMEN
Ante la nueva realidad de la Convergencia Europea en torno a la educación, títulos y perfiles profesionales, la formación basada en las competencias se convierte en el componente principal de la enseñanza universitaria. La capacitación de profesionales demanda una evolución en la preparación propiciada, sobretodo en esta última década, por las transformaciones tecnológicas, económicas y sociales que están generando cambios profundos en los contenidos, medios, métodos y formas sociales del trabajo.

En este artículo se analiza uno de los más importantes retos que han de afrontar las universidades en los próximos años: redefinir su oferta formativa tratando de dar una respuesta más ajustada a las nuevas demandas que tanto la sociedad como los individuos le reclaman. Trataremos de explorar un modelo de formación basado en las competencias y los cambios que dicho modelo implica para el profesorado y el alumnado. Nos situamos en el marco de los procesos de innovación como actividad colaborativa.
\end{abstract}

Palabras clave: Educación superior, procesos de innovación, competencias.

\section{ABSTRACT}

Given the new reality of the European Higher Education Area (EHEA), professional titles and profiles, formation based on competences has become the main component of formation at the university. In this last decade, technological, economic and social transformations are placing professional demands on revising the content, means, methods and social forms of work.

This article analyzes one of the most important challenges that universities have to confront in the coming years: to redefine their formative offer to provide a more fitting answer to society's and individuals' demands. We will explore a model of formation based on competences and the changes that this model im- 
plies for teaching staff and students. Reflections are placed within a framework of the innovation processes. Innovation is suggested as a collaborative solution.

Key words: Higher education, processes of innovation, competences.

\section{Delimitación conceptual}

Pero ¿por qué hablamos de competencias profesionales? La literatura sobre competencias profesionales es profusa y difusa (Bohereham, 2004; Le Boterf, 2001; Lévy-Leboyer, 1997; Mertens, 1996; Prieto, 1997; Zarifian, 2001), por lo divergente y complementaria. Unos autores establecen tres tipos de clasificaciones conceptuales de competencia: unidimensional, multidimensional e integradora (Rodríguez, 2006). Otros, proponen una clasificación para el enfoque analítico de las competencias: conductista, funcionalista y constructivista (Blanco, 2007). Así que resulta complejo establecer unas líneas de base de las que partir.

Antes de proceder a presentar una aproximación a la definición del concepto de competencias profesionales se considera necesario realizar una breve introducción acerca de la naturaleza compleja, cambiante y polisémica del término competencia: ¿Es una nueva palabra de moda que no se corresponde con un concepto nuevo? ¿ $\mathrm{O}$ un enfoque original que se ha hecho necesario por la evolución del trabajo? En este caso, ¿cuáles son las relaciones de las competencias con los conocimientos? ¿Y con las aptitudes? ¿Y con la personalidad?

Así, el conocimiento de estos componentes básicos se convierte en pieza clave para el desarrollo de las diversas propuestas formativas capaces de responder con eficacia a dichos requerimientos y adaptarse a los cambios venideros (Tejada, 1999) y es que, como este mismo autor plantea, ningún experto pone en duda que las competencias van más allá de la mera especialización técnica para incorporar dimensiones relacionales y sociales. De acuerdo con todo ello se puede sintetizar que (Tejada, 1999:1):

1. El concepto de competencias comporta todo un conjunto de conocimientos, procedimientos y actitudes combinados, coordinados e integrados.

2. Las competencias sólo son definibles en la acción.

3. La experiencia se muestra ineludible.

4. El contexto es clave.

Es decir, la competencia implica dominio, posesión de capacidades sin las que es imposible llegar a ser competente. Sin embargo "ser capaz" no significa lo mismo que "ser competente". Las competencias no se pueden reducir ni al "saber" ni al "saber hacer" sino a la movilización de estos recursos. En el proceso dinámico de construcción de las competencias no es suficiente el proceso de capacitación. El concepto de competencia es indisociable de la noción de desarrollo o de aprendizaje continuo.

El concepto de competencia comenzó a ser utilizado como resultado de las investigaciones de David McClelland en los años 70, las cuales se enfocaron en la identificación de las variables que permitieran explicar el desempeño en el trabajo. De hecho, un primer hallazgo lo constituyó la demostración de la insuficiencia de los tradicionales tests y pruebas para predecir el éxito en el desempeño laboral. 
McClelland y Spencer (1994) lograron confeccionar un marco de características que diferenciaban los distintos niveles de rendimiento de los trabajadores a partir de una serie de entrevistas y observaciones. La forma en que describieron tales factores se centró más en las características y comportamientos de las personas que desempeñaban los empleos que en las tradicionales descripciones de tareas y atributos de los puestos de trabajo.

A continuación, recogemos varias definiciones sobre el concepto de competencia ya que, conforme avanzan los diferentes acercamientos y nuevas explicaciones, se diversifican los conceptos sobre competencia.

- El Diccionario de la Real Academia de la Lengua (1995: 49) define competencia como pericia, aptitud, idoneidad para hacer algo o intervenir en un asunto concreto.

- Bunk (1994: 99) define la competencia como:

El conjunto de conocimientos, procedimientos, actitudes y capacidades que una persona posee y son necesarias para afrontar de forma efectiva las tareas que requieren una profesión en un determinado puesto de trabajo, con el nivel y calidad de desarrollo requeridos; resolver los problemas emergentes con iniciativa, autonomía y creatividad; y adaptarse al entorno sociolaboral y colaborar en la organización del trabajo.

- Según Levy-Leboyer (1997: 54):

"Las competencias son repertorios de comportamientos que algunas personas dominan mejor que otras (...). Estos comportamientos son observables en la realidad cotidiana del trabajo (...). Ponen en práctica, de forma integrada, aptitudes, rasgos de personalidad y conocimientos adquiridos (...) las competencias representan la unión entre las características individuales y las cualidades requeridas para llevar a cabo misiones profesionales precisas.

- Ibarra (2000: 102) la define como la capacidad productiva de un individuo que se define y mide en términos de desempeño en un determinado contexto laboral, y no solamente de conocimientos, habilidades o destrezas en abstracto; es decir, la competencia es la integración entre el saber, el saber hacer y el saber ser.

- En el borrador de directrices para la elaboración de títulos, el MEC (2006. Apdo. 15) define las competencias como una combinación de conocimientos, habilidades (intelectuales, manuales, sociales, etc.), actitudes y valores que capacitarán a un titulado para afrontar con garantías la resolución de problemas o la intervención en un asunto en un contexto académico, profesional o social determinado.

Aunque estamos lejos de poder ofrecer una definición única del término competencia, estas serían algunas de las características que podemos extraer de las definiciones dadas:

- Ser competente implica poder realizar una actividad profesional, resolver problemas o desarrollar proyectos en tiempo oportuno movilizando integralmente un saber realizar, conjuntamente con los saberes conceptuales pertinentes y con capacidades diversas de acción y de relación, con el fin de obtener resultados de calidad. 
- Implica conocer lo que se realiza, tener conciencia de sus consecuencias y capacidad de evaluar la acción. Supone capacidad para aprender, innovar y generar nuevos conocimientos.

- Otra característica de las competencias es su capacidad de transferibilidad hacia otras situaciones o procesos de acción. No es un saber válido exclusivamente en tareas específicas o rutinarias. La fortaleza de la formación basada en competencias reside en la posibilidad de ampliación de los saberes más allá de la simple ejecución de tareas delimitadas. En este sentido, ser competente supone la capacidad de ver, analizar e intervenir en un mundo complejo en el que se interrelacionan aspectos estrictamente técnicos con aspectos culturales, sociales, éticos, políticos y tecnológicos.

En resumen, actualmente se entiende por competencia un conjunto integrado de conocimientos, capacidades, representaciones y comportamientos movilizados para resolver problemas profesionales. El concepto de competencia implica un saber hacer complejo resultado de la movilización, integración y adecuación de conocimientos, habilidades y actitudes, utilizados eficazmente en diferentes situaciones.

En este artículo se examinan algunos de los cambios necesarios que plantea el nuevo modelo formativo centrado en las competencias. En la segunda parte, exploraremos las demandas de este enfoque sobre los cambios de rol del profesorado y las nuevas exigencias con las que se tendrá que enfrentar el alumnado. El artículo concluirá con algunas dificultades en el camino de implantación de este modelo de formación y algunas propuestas de intervención para hacerlo factible.

\section{La formación en competencias en el ámbito universitario}

En este apartado explicaremos como surge el enfoque basado en competencias en los estudios superiores.

En el ámbito universitario este modelo está en la base del proceso europeo de convergencia hacia un Espacio Europeo de la Educación Superior (EEES) y el proyecto piloto Tuning (González y Wagenaar, 2003). Este proyecto se llevó a cabo por las universidades de Deusto y Groningen, con el patrocinio de la Comisión Europea y la colaboración de 70 universidades europeas. Su objetivo era el diseño de una metodología para la comprensión del currículo que lo hiciera comparable. Como parte de la metodología se introdujo el concepto de resultados del aprendizaje y competencias.

El proyecto centra su enfoque de competencias en el proceso de aprendizaje y por lo tanto focaliza el diseño formativo en el trabajo del estudiante. Entendemos que aquí se encuentra el eje central por el que se hacen necesarios toda una serie de cambios. Como consecuencia, el profesorado universitario, adopta un rol de orientador y guía del proceso de aprendizaje del alumnado dejando de ser el centro del acto educativo.

En el Proyecto Tuning las competencias representan una combinación dinámica de atributos -con respecto al conocimiento y su aplicación, a las actitudes y a las responsabilidades- que describen los resultados del aprendizaje de un determinado programa, o cómo los estudiantes serán capaces de desenvolverse al finalizar el proceso educativo. 
El proyecto propugna una formación basada en competencias generales que deben plantear los planes de estudio y específicas, que desarrollen la titulación y, por ende, la profesión, esto es, capaz de combinar elementos teóricos y procedimentales, conocimientos disciplinares y aprendizajes prácticos, desarrollo científico y formación en actitudes y valores.

Esta incorporación seguramente ha estado influida por el amplio uso del término competencias en el ámbito laboral y el deseo de unir la Universidad con el mundo profesional posterior con que el egresado ha de enfrentarse tarde o temprano. El trabajo es la actualización, la realización de una competencia.

La sociedad moderna delega en la educación superior la función de desarrollar en los estudiantes las competencias que les permitan actuar de manera eficaz en dicha sociedad (Yániz y Villardón, 2006). El perfil de egreso de cada titulación define las características deseadas en la persona titulada para desenvolverse de la mejor manera posible como profesional y como ciudadano. El perfil profesional define las competencias profesionales que permiten desempeñar adecuadamente sus funciones, y el perfil ciudadano supone un "estar en la sociedad" de una forma preactiva comprometida con la mejora de la misma y el desarrollo personal propio y de los demás. Este perfil de egreso, por tanto, empieza a ser un referente fundamental de la formación universitaria.

La orientación de la formación universitaria está suscitando, igualmente, un fuerte debate sobre la relación entre formación y empleo (carreras orientadas al empleo o a la formación general de los sujetos).

Estamos convencidos de que en el mundo contemporáneo la formación debe diseñarse partiendo de las competencias profesionales necesarias en el nuevo contexto europeo y de las nuevas realidades sociales que están requiriendo (Mora, 2003), a todas luces, profesionales que posean las competencias laborales necesarias para hacer frente a estas transformaciones.

Un modelo de enseñanza universitario basado en las competencias apunta a la formación no sólo como la adquisición de conocimientos y habilidades específicas, sino también en las actitudes y hábitos. Formar a los universitarios supone algo más que proporcionar conocimientos. El objetivo formativo, por tanto, supone la adquisición de conocimiento, el desarrollo de habilidades y la capacidad de aplicar estos recursos de forma adecuada a cada una de las situaciones que se presenten.

En este proceso, va a tener especial importancia va a tener la adaptación de los programas antiguos por objetivos (en muchos casos sólo programas de contenidos disciplinares) a programas por competencias y la subordinación de los contenidos disciplinares a dichas competencias que, conceptualmente, son un "saber hacer complejo e integrador" (Lasnier, 2000), lo que implicará un modo absolutamente distinto de organización curricular, al mismo tiempo que un cambio sustancial en los métodos de enseñanza y aprendizaje que, en esta nueva situación, pasan de ser generalmente centrados en el profesor a tener que centrarse en los estudiantes, buscando situaciones de aprendizaje contextualizadas, complejas, focalizadas en el desarrollo en los estudiantes de la capacidad de aplicación y resolución de problemas lo más reales posibles. El contenido disciplinar será el vehículo para plantear diferentes estrategias de aprendizaje y enseñanza 
que logren la integración del conocimiento teórico, es decir, el qué, con el cómo (conocimiento procedimental) y el por qué (conocimiento condicional, contextualizado).

Así pues, la introducción de las competencias como objeto de la formación universitaria lleva a una revisión del propio concepto de formación. La formación es algo más que la mera información. Formar no es transmitir. La formación no es sólo acumular conocimientos. Las competencias hablan de conocimiento aplicado. El aprendizaje de competencias supone conocer, comprender y usar pertinentemente (De la Cruz, 2003). Como teoría explicativa de este aprendizaje, el modelo conductista se queda corto. La explicación cognitiva y constructivista del aprendizaje es más coherente con la naturaleza de las competencias. Desde esta perspectiva las exigencias del aprendizaje eficaz propuestas por este enfoque se caracterizan por ser un proceso constructivo, activo, contextualizado, social y reflexivo. Aprender con sentido, aprendizaje significativo, a partir de lo que se conoce, activo y con tareas reales, serán las garantías de un aprendizaje duradero.

A continuación explicitaremos algunos de los cambios de rol que se derivan de este modelo tanto para el profesorado como para el alumnado.

\section{Cambios en el rol del profesorado}

Incitar a este proceso no es tarea fácil. En este nuevo enfoque, la planificación juega un papel fundamental. Como afirma De Miguel (2003:19): La calidad de la enseñanza no depende tanto de lo que el profesor "sabe" como de lo que "planifica" como objetivos de la materia en función de las necesidades y posibilidades de sus alumnos y lo que "hace" para ofrecer a todos los estudiantes oportunidades para el aprendizaje.

La tarea fundamental del profesor es enseñar al estudiante a aprender a aprender, ayudar al alumno en la creación de unas estructuras cognitivas o esquemas mentales que le permitan manejar la información disponible, filtrarla, codificarla, categorizarla, evaluarla, comprenderla y utilizarla pertinentemente. En definitiva, preguntarse cómo formar en competencias es preguntarse cómo organizar y gestionar los procesos de aprendizaje.

Este nuevo enfoque en la formación necesita invariablemente un cambio en el rol del profesorado que no va a ser fácil y tiene muchas implicaciones (Zabalza, 2007). A continuación pasaremos a describir alguna de ellas.

Asistimos en feliz expresión de Martín (1989: 69) a un supermercado del conocimiento. Hasta hace relativamente poco tiempo, las escuelas y las universidades eran los centros fundamentales del saber. Hoy en día, la información es multidireccional (Villa, 1997), procede y se expande desde múltiples fuentes. Esa información, se extiende a través de variados canales como prensa, televisión, radio, CD-rom, redes informáticas, etc. Todo ello, da pie a la reflexión general sobre el acceso al conocimiento y su universalización, donde la cantidad de la misma es un obstáculo que provoca junto con la cantidad de tiempo para analizarla detenidamente, un consumo desmedido y acrítico. El problema más grave que deberá resolver la educación es cómo promover el deseo de saber frente a la sobreinformación circundante en la sociedad y cómo formar los marcos de referencia para procesar la información disponible. De ahí que sea indispensable 
que los sistemas educativos puedan formar para manejar y orientar esas técnicas" (Tedesco, 1997: 154).

Por ello, consideramos fundamental entre las tareas del profesorado la formación para la comprensión y la selección adecuada de la misma. Uno de los principales cambios que se nos exige a los profesores es dejar de ser tanto una fuente de información para pasar a tener un papel como facilitadores del conocimiento. Las tendencias señaladas hace ya unas décadas siguen manteniéndose vigentes, aunque se muestran más urgentes. Así, por ejemplo, Goble y Porter (1980: 55) afirmaban que: "Los nuevos conocimientos son descubiertos constantemente a un ritmo tan acelerado que apenas pueden ser codificados adecuadamente, y menos aún poseídos... La noción del profesor como quien posee un depósito fijo de conocimientos determinados que parecen útiles es cada vez menos sostenible. Efectivamente en algunas materias como informática, los contenidos se quedan anticuados en el mismo curso académico en el que se han tratado. De cara a un futuro próximo, se calcula que las personas tenderán a realizar durante su vida laboral un aprendizaje profesional equivalente a cinco carreras universitarias".

Entendemos que la relación que se nos pide a los docentes con respecto al alumnado es diferente, pasar de la función de único informador a la de acompañante, guía y orientador, convirtiéndose ya no tanto en el que imparte los conocimientos como en el que diseña nuevas situaciones de enseñanza-aprendizaje y ayuda a los alumnos/as a encontrar, organizar y manejar esos conocimientos. Los alumnos/as se convierten en investigadores provistos con estos nuevos instrumentos. Los docentes enseñan a los alumnos a evaluar y a tratar práctica y críticamente las informaciones que tienen a su alcance. Esta manera de proceder resulta mucho más próxima a la vida real que los métodos tradicionales de enseñanza.

La finalidad de sus funciones docentes no será transmitir conocimientos sino orientar de forma individualizada para crear alumnos emprendedores que sepan y puedan generar su propio trabajo como profesionales autónomos. Como indica Bain (2005) el buen profesor se preocupa no solamente por lo que dice/escuchan sus alumnos sino, también y mucho, por averiguar lo que retienen de todo lo oído así como, y de modo especial, por el uso que hacen de ello en su vida cotidiana, es decir, por la efectividad educativa.

Como apunta Biggs (2006) formar desde la perspectiva de las competencias demanda un cambio sustancial en el rol del profesorado que podemos denominar "del yo al nosotros". Esto implica desarrollar culturas de trabajo colaborativo o "comunidades de aprendizaje profesional». La potenciación de los procesos cooperativos exige la creación de una nueva cultura que rompa con el esquema de individualización y balcanización aún vigente. La búsqueda de centros educativos de calidad parte de la convicción de que es posible crear, conservar y transformar la cultura, a pesar de que se requieran tiempos largos, para adecuarla a las nuevas exigencias. Este autor entiende que el hecho de compartir concepciones y convicciones sobre la enseñanza y el papel de los profesores es fundamental para lograr acciones coordinadas y de calidad.

Los equipos de profesores suelen identificarse como estructuras a las que se reconoce un elevado potencial de mejora y capacidad de cambio. Sin embargo, la práctica docente muchas veces se ha caracterizado de individualista y celular, donde hay "zonas acotadas" con pocas posibilidades de compartir recursos e ideas y de intercambiar ex- 
periencias. Pero, además de ser ineficaz la organización para los estudiantes, al proporcionar una formación fragmentaria y contradictoria, también lo es para el profesorado, al dificultar un trabajo colaborativo que facilite el aprendizaje mutuo. Es una actuación en equipo tanto por la magnitud como por las características y exigencias de la tarea. Incluye partes de trabajo común entre distintos profesores y la coordinación de programas y actuaciones individuales" (Yániz y Villardón, 2006: 78). Tradicionalmente en la universidad ha existido una práctica de libertad de cátedra. Es preciso clarificar, por tanto, que el trabajo colaborativo no se opone a la autonomía del profesor si se orienta adecuadamente. De hecho, se trata de conjugar el nivel de estructuración y el grado normativo para que nadie se sienta coartado en sus iniciativas.

Desarrollar un proyecto formativo integrado y complejo, como es un plan de estudios universitario, que aspire a proporcionar una formación integral y la adquisición de un conjunto sólido de competencias que configuren un perfil de referencia, solo puede alcanzarse con la articulación y coordinación de todo el profesorado implicado. Además, compartimos la idea de que "la organización de un currículum abierto a las demandas de la sociedad actual requiere la cooperación de varias especialidades académicas. El «blindaje» de los planes de estudio hiperespecializados constituye un error estratégico, aparte de una irresponsabilidad" (Quintanilla, 1998:93).

Transformar los planes de estudio diseñados a partir de programas estancos elaborados por cada profesor, en proyectos integrados elaborados por un equipo supone una innovación profunda del currículo universitario que incide en la cultura y probablemente en la estructura de la universidad. El proceso de cambio necesita una planificación específica a medio y a largo plazo, que proporcione apoyo y cobertura a la planificación docente.

Se trata de una visión de la enseñanza en la que el alumno/a es el centro o foco de atención y en la que el profesor/a juega, paradójicamente, un papel decisivo. DesarroIlar procesos docentes colaborativos significa, por tanto, que las materias están vinculadas no sólo a un profesor sino a un conjunto de profesores. Es más, supone tomar conciencia de que la aportación de una materia debe estar en relación con el conjunto del plan de estudios y que éste, a su vez, debe estar desarrollado en base a las competencias profesionales futuras que el marco de una titulación concreta.

Se trata del paradigma de la colegialidad al que también Lesourne (1993) apunta. Esta vez se trata de la siempre difícil tarea del trabajo en equipo. En un interesante informe sobre la educación y la sociedad (Informe Bricall, 2000: 44) se afirma que la profesión docente "no se define como la prestación tarifada de una actuación individual del tipo de los profesionales liberales, sino como la participación en un aparato de producción, con una devolución de la responsabilidad resultante en la participación de tareas concertadas". Se abandona la concepción de la docencia como tarea individual. Continúa Lesourne (p. 67), "esta modalidad supone individuos autónomos, pero dentro de equipos con un sentido de responsabilidad colectiva".

Lo más importante de nuestra acción docente es la coordinación con el resto de nuestros compañeros, la programación de la acción educativa, la elaboración de materiales, la selección significativa de contenidos de cara al tipo de profesional que desea- 
mos formar, la formación permanente entendida como reflexión de nuestra propia práctica, el intercambio de experiencias educativas y el trabajo en equipo.

Por todo ello, es importante desarrollar la cultura del trabajo en equipo. El Ilamado paradigma de la colegialidad parte de la concepción de las áreas departamentales como unidades funcionales de planificación, de acción, evaluación, cambio y formación. Comprobamos, no sin dificultad, que las ventajas del planteamiento colegiado son notables:

a) Multiplica la eficacia al incidir todos los profesores en las mismas ideas, en las mismas actitudes, en los mismos objetivos y valores.

b) Permite subrayar lo que es importante y dejar al margen lo que es baladí.

c) Ayuda a eliminar los errores, las repeticiones innecesarias, las omisiones de aspectos importantes.

d) Permite que los profesores aprendamos unos de otros, que nos intercambiemos materiales, ideas, experiencias y que nuestra formación continua se dé en la propia práctica, no tanto a través de cursos, conferencias o lecturas cuanto a través de una reflexión sistemática, rigurosa y compartida sobre la práctica (Shön, 1983, 1987). La reflexión de los grupos de trabajo en torno a problemas y peculiaridades de la enseñanza son caminos de perfeccionamiento y desarrollo profesional (Fullan, 1988). El trabajo en grupo resulta potencialmente eficaz para el aprendizaje.

e) Favorece el desarrollo de actividades pedagógicamente enriquecedoras como el intercambio, la ayuda del compañero y la profesionalización docente.

f) Aumenta la coherencia de los planteamientos educativos.

La planificación conjunta de la enseñanza, la acción preparada en conjunto y cuidadosamente realizada y el análisis compartido de la misma se convierten en una forma eficaz de comprensión de la práctica y de mejora de la racionalidad de la misma.

Este enfoque de la docencia, nos insta a la formación permanente ya que como docentes necesitamos de una serie de cualidades y competencias que hacen más compleja y diversificada nuestra función docente como mediador, facilitador del aprendizaje, asesor y tutor, capaz del trabajar en equipo, creador de materiales didácticos, innovador en la docencia, usuario de nuevas tecnologías, etc. todo ello sin perder de vista nuestra condición de formadores y de potenciar la autonomía intelectual y la responsabilidad social de los estudiantes.

\section{Cambios en el rol del alumnado}

Como mencionábamos, entre los objetivos explícitos del proceso de Convergencia Europea basado en el enfoque de las competencias está el que el estudiante cobre un perfil más destacado en su proceso de aprendizaje. El sistema, diseñado conforme a la filosofía ECTS (European Credit Transfer System), convierte al "crédito" en la unidad básica de planificación, de actuación y de evaluación.

Esta nueva unidad de medida comprende todas las actividades que el estudiante realiza fuera y dentro del aula para conseguir el aprendizaje. O dicho de otro modo, se 
centra en el aprendizaje de los estudiantes que se convierten en protagonistas de su propio proceso. Lamentablemente, hasta ahora el sistema universitario giraba en torno al trabajo docente y en la clase presencial, sin destacar el enfoque orientado al trabajo del alumnado. Para que esto sea posible es necesario el "Shift from teaching to learning" (Jarvis, 2006). Prosperar en la línea europea que considera el crédito europeo como la productividad del estudiante, significa ir modificando gradualmente la orientación magistral hacia un enfoque de mayor autonomía y compromiso académico del alumnado, lo que implica un cambio en el trabajo del estudiante.

El interés en el desarrollo de competencias en los programas educativos coincide con un enfoque de la educación centrado principalmente en el estudiante y en su capacidad de aprender, que exige más protagonismo y cotas más altas de responsabilidad puesto que es el estudiante quien debe desarrollar la capacidad de trabajar con la información, buscarla y valorarla en un modo más diverso (biblioteca, profesores, Internet, etc.). Los estudiantes tienen, además, una mayor familiaridad con las TIC y por ello son capaces de utilizarlas con mayor facilidad y provecho. Los nuevos sistemas informáticos y la nueva concepción de su rol conllevan que su actividad empieza antes de la clase presencial y continúa después de ella. El alumno ha de ser capaz de transformar el uso de TIC de prácticas convencionales en procesos de aprendizaje significativo.

Hasta ahora, el enfoque tradicional se ha centrado en la acumulación de la mayor cantidad de conocimientos posible, pero en un mundo rápidamente cambiante esto no es relevante. La "sociedad del conocimiento" es también una "sociedad del aprendiza$\mathrm{je}^{\prime}$ ". La formación para la Sociedad del Conocimiento requiere acciones educativas relacionadas con el uso, selección, utilización y organización de la información, de manera que el alumno vaya formándose como un maduro ciudadano de la sociedad de la información. Con lo que los estudiantes pasan a ser el eje del nuevo sistema enseñanzaaprendizaje, orientado a facilitarles el acceso a una formación integral que les facilitará su inserción laboral. Esta idea está íntimamente ligada a la comprensión de toda educación en un contexto más amplio: el aprendizaje continuo a lo largo de toda la vida, donde el individuo precisa ser capaz de manipular el conocimiento, de ponerlo al día, de seleccionar lo que es apropiado para un contexto específico, de aprender permanentemente, de entender lo que se aprende, de tal forma que pueda adaptarlo a nuevas situaciones que cambian rápidamente.

Este punto de vista pone al estudiante en el centro del proceso como "constructor de su propio conocimiento", concibiéndolo en un sentido amplio, esto es, tanto referido a conceptos, como a procedimientos y actitudes. Por consiguiente, afecta la manera de guiar las actividades educativas y la organización del conocimiento, que pasan a ser regidos por las metas del estudiante.

Por un lado, el trabajo cooperativo y los trabajos en grupo cobran un papel destacada en este proceso. Por otro, la acción tutorial. Para hacer posible esta nueva dinámica educativa, los estudiantes ha de desarrollar la habilidad para diagnosticar las propias necesidades, planificar, crear las condiciones para alcanzar los propios objetivos, etcétera, evaluando al tiempo la efectividad de la actividad educativa.

Se requiere, en definitiva, introducir en nuestro nivel de enseñanza otro estilo, una mayor flexibilidad que busque la potenciación en los estudiantes y su capacidad para 
actuar en el mundo real. Ello lleva a poner el acento en lograr que el alumnado adopte una concepción flexible, fiable y constructiva del conocimiento que se enseña en la universidad. Los estudiantes deben aprender a ser capaces de construir sus propios conocimientos a partir de los contenidos de las diferentes materias de manera significativa y profunda, permanente y, sobre todo, generalizable. La institución universitaria ha de formar estudiantes, y futuros profesionales, estratégicos y autónomos (Monereo y Pozo, 2003), habilitados para responder de manera eficaz y diligente a los cambios y versiones que les ofrezcan los contextos en los que interactúan.

En este marco cobra relevancia a la idea de "participación" de los estudiantes, en el sentido, por una parte, de aprovechar mucho el "tiempo de clase", no tanto para dictar o explicar contenidos, como para analizar, debatir y contrastar sus "ideas previas" con contenidos e ideas que se les ofrece o que en ocasiones surgen espontáneamente de otros compañeros. Esta dimensión de "participación" también debe servir para que el alumnado se habitúe a expresar oralmente sus ideas (para lo cual debe previamente organizarlas) y para propiciar situaciones variadas de comunicación (diálogos, debates, etc.).

Trabajando desde esta perspectiva también hemos de ser conscientes, en principio, de algunas dificultades por parte de los estudiantes quienes familiarizados con la tradicional "clase magistral" pueden sentirse incómodos durante algún tiempo. En este instante el papel del profesorado es convencerles de que son agentes de su propio aprendizaje. También quieren saber lo que realmente tienen que hacer para aprobar y el número de páginas que necesitan saber para los exámenes. Por otro lado, siempre hay determinados estudiantes que tienen más dificultades para coordinarse con sus compañeros y generar un trabajo en equipo. A continuación subrayaremos algunas de estas dificultades en este proceso de transición de un modelo a otro.

\section{Algunas dificultades ante el cambio y reflexiones finales}

Como era de esperar no todo son vítores en este cambio de enfoque. Este proceso no está al margen de las dificultades que se presuponen a todo tipo de cambios. Es algo con lo que hay que contar ya que no es lo mismo acomodarse a un cambio técnico que a un cambio de mentalidad o de actitud.

Constatada la necesidad de un cambio de paradigma con toda la dificultad que ello conlleva quedaría pendiente una tarea también compleja de decidir cuáles son las competencias genéricas en las que deberíamos insistir desde la Universidad. En este sentido (Corominas, Tesouro, Capell, Teixidó, Pèlach y Cortada, 2006) han realizado un interesante trabajo en el que se exploran distintas categorías propuestas por Universidades europeas, canadienses y americanas, al mismo tiempo que ofrecen vías de intervención para incorporar las competencias en la formación universitaria.

A veces, aflora el escepticismo respecto a la posibilidad de mejorar la calidad de la formación o el pesimismo referido al cambio en profundidad necesario que hace, que unas veces unos otras veces otros, prefieran mantener la rutina institucionalizada. En otras ocasiones, son causas externas las que pueden echar para detrás. La cultura nunca es totalmente aceptada ni totalmente rechazada; nunca terminan las fuerzas dominantes de instituirse y tampoco los elementos contestatarios generan el cambio radical y simultáneo de los rasgos que definen una cultura. Puede afirmarse entonces que, en la orga- 
nización, sus componentes culturales están en continuo movimiento y son el resultado de una síntesis de antagonismos. Pero esto también nos lleva a recordar que si en toda organización la cultura nunca es homogénea, y hay un permanente conflicto, la inestabilidad, la crisis y el cambio se está dando de hecho ya en toda organización.

Entre el profesorado e incluso entre las autoridades universitarias existen los entusiastas y eufóricos, así como los escépticos. Es decir, los que creen que nada va a cambiar ni puede cambiar en una institución milenaria como es la Universidad y los que afrontan la convergencia como una oportunidad de revisar y mejorar lo que estamos haciendo y cómo lo estamos haciendo.

En un momento como el presente, en el que vivimos la "moda" de la calidad total, suscitada por la Administración, como impulso de cambio en las organizaciones educativas, es necesario cuestionarse a qué tipo de corriente responde esta idea de cambio; si esta "ideología" favorece verdaderamente una "cultura de calidad" en dichas organizaciones o si lo que puede estimular es una "cultura de la apariencia" que responda frívolamente a una imagen exigida por la administración, pero que sigue conservando en el fondo "lo mismo de siempre", puesto que todo cambio promovido desde fuera no modifica las bases de la organización.

Pero no hay cambio organizativo en profundidad, que no implique un cambio cultural, puesto que todo cambio serio afecta a las raíces mismas de la organización. "Un cambio en la organización supone un cambio de cultura, en definitiva un cambio de pensamiento de los miembros de la comunidad educativa. Se ha de plantear desde el punto actual de la organización, a través de pasos concretos con la comprensión y participación de todos" (Domenech y Viñas, 1997: 83).

Todo cambio cultural, es de por sí un proceso muy lento puesto que es un elemento de contenido ideológico elaborado a lo largo de los años y que afecta a la filosofía básicas de la organización. Cambiar las conductas supone cambiar los valores que subyacen y eso sólo se puede realizar a través del autoconvecimiento y por tanto de la reflexión sobre aquello que conviene cambiar. Una verdadera innovación se caracteriza por trascender a los sujetos para incidir en la cultura institucional hasta que esa mejora propuesta forma parte de la misma (Hannan, 2006). Es decir, se consolida o institucionaliza.

En la complejidad que requiere este cambio entendemos que el profesorado juega un papel fundamental. Hará falta preparar al profesorado para asumir este reto. Los cursos de formación en este sentido son cruciales, pero lo que entendemos que puede conllevar un verdadero cambio son los procesos colaborativos de equipos de profesores que reflexionan sobre la propia práctica, que planifican conjuntamente y que evalúan los resultados de sus acciones.

Sin duda, existen otros factores añadidos que dificultan el cambio cultural y que van a influir poderosamente en todo proceso de cambio. Serían los siguientes:

- El tiempo: se necesitan muchos años para lograr un cambio fundamental en la cultura de una organización.

- La fuerza de la cultura que se quiera cambiar: si la cultura o la subcultura anterior ha dado respuesta y sentido coherente a los miembros de la organización durante 
años, se sentirán reacios a abandonarla para aceptar otra. Muchos de sus componentes se habrán convertido en elementos "sagrados" cuyo análisis, cuestionamiento y posible cambio serán muy discutidos. Económicamente cuesta mucho, pues requiere invertir tiempo, esfuerzo humano, asesoramiento adecuado y recursos organizativos.

- Lo atractivo que ese cambio les resulte a todos los miembros de la organización: cualquier propuesta con la que todo el mundo sale ganando se acepta más fácilmente que las propuestas en donde algunos tienen que perder. Igualmente hay que tener en cuenta que la dificultad del cambio se ve incrementada porque dentro de una organización no hay sólo una cultura, sino diversas subculturas, y muchas veces en permanente conflicto: ¿cuál debería cambiar?, ¿o tendrían que cambiar todas?, ¿estarían todas de acuerdo en cambiar?

El discurso que hemos planteado hasta ahora nos ha puesto de manifiesto que ante los cambios sociales, culturales, económicos, humanos en definitiva, la universidad no puede esconder la cabeza y esperar a sobrevivir como institución sin adaptarse a las circunstancias actuales. Las universidades están hoy por hoy, más que nunca, expuestas al cambio y a la incertidumbre.

Si algo hemos aprendido de las innovaciones educativas es que su dificultad es proporcional a su complejidad. Llevar a cabo una experiencia innovadora en el aula resulta relativamente fácil, pero diseñar un proyecto con cambio de fines y roles en los que se comprometan varias personas resulta mucho más complicado.

Una de las lecturas o interpretaciones que se puede hacer es que existe una sensibilización sobre la necesidad de que las cosas sean diferentes a como son. Sin embargo, la decisión de emprender el cambio ha venido desde fuera. El éxito de la mejora de la universidad implica adaptar cambios externos a propósitos internos.

También es importante que la mejora de la universidad afecte a todos los "niveles", puesto que, fruto de la investigación y la experiencia, sabemos que el cambio no tendrá éxito si no afecta a todos los niveles de la organización.

A pesar de las dificultades nos debe animar el deseo de superar los paradigmas didácticos en los que a veces nos encontramos en la universidad moviéndonos entre los discursos teóricos y la práctica profesional:

- No es coherente mantener el discurso teórico sobre la enseñanza activa y participativa mientras se centra la metodología en las clases magistrales (muchas veces útil) impartiéndolas de manera magistral.

- No es coherente desarrollar teorías sobre el aprendizaje colaborativo mientras en la organización prevalece el individualismo de la actuación de cada uno como profesor que defiende su libertad de cátedra.

- No es coherente alimentar un discurso sobre el profesor como investigador mientras la práctica se asienta en prácticas que favorecen la repetición y la memoria.

- No es coherente proponer la mejora continua desde la innovación como camino de avance si nuestra comunidad educativa no se identifica como un colectivo de profesores que aprenden. 


\section{Conclusiones y propuestas de intervención}

El principio fundamental que sostiene este modo de desarrollar la enseñanza basada en las competencias establece que los estudiantes han de ser protagonistas de su propio proceso de aprendizaje, de tal modo que el profesor indicará los objetivos y actuará como orientador y mediador del mismo. Esta nueva filosofía se contrapone a la tradicional, en la cual la enseñanza del profesor era lo primordial. Por el contrario, desde este enfoque se insiste sobre todo en el aprendizaje de los estudiantes; lo importante es lo que hace o puede hacer el estudiante; en cierta medida se está hablando de una enseñanza más individualizada, de sus capacidades e intereses.

Reconociendo las dificultades que conlleva este cambio, pensamos que este enfoque puede ser una gran oportunidad que tienen las universidades europeas para intentar el cambio, lo que requiere una modificación de la cultura en la universidad como organización, en sus estructuras e infraestructuras. Un cambio de paradigma que implica transformar hábitos y costumbres de profesores y estudiantes, y un cambio en la propia sociedad, que debe entender el esfuerzo que esto va a conllevar y los recursos extras que las universidades requerirán para hacer que el cambio sea viable.

El enfoque de "competencias" en el ámbito universitario europeo está ligado al deseo de un desarrollo integral de los estudiantes basado en la adquisición y desarrollo de sus habilidades, actitudes y valores, así como a un conocimiento que pueda ser transferible a las diversas situaciones laborales, profesionales y sociales en las que puede verse inmerso.

Teniendo todo esto en cuenta nos atrevemos a formular un planteamiento aproximativo de lo que deberían ser los principales elementos de intervención para posibilitar la puesta en marcha de este enfoque.

En primer lugar, conseguir la traducción en términos de competencias y aplicado a la planificación y a la práctica cotidiana del profesor significa que es necesario llegar a un consenso sobre el significado y alcance de las competencias en el ámbito educativo. En cuanto a las competencias específicas, la dificultad estriba en dotar de contenido a muchas de dichas competencias y en saber cómo se pueden proyectar sobre la actividad formativa de cada centro universitario. Esto no es fácil, entre otras cosas por las aristas epistemológicas, la amplitud polisémica del mismo concepto y por las dificultades de los contextos en donde se les inscribe. Si las competencias son capacidades para desarrollar una determinada actividad, su adquisición y desarrollo debe hacerse mediante el ejercicio de metodologías de aprendizaje más activas y recursos apropiados.

En segundo lugar, estas nuevas tareas exigen a los profesores actuales un esfuerzo añadido de formación continua en contextos específicos de su especialidad. Los profesores necesitan hacerse expertos en planificación y desarrollo de programas. Deben aprender a planificar por competencias que le permitan prever, organizar y reformular los procesos de enseñanza-aprendizaje en función de sus efectos. Para ello, sería conveniente que las universidades dispusieran la oferta de cursos teórico-prácticos, seminarios, debates, experiencias y proyectos de formación docente y apoyaran iniciativas de los departamentos en este sentido; actividades formativas que deberían estar convenientemente acreditadas, como cualesquiera otras. 
En tercer lugar, en este contexto, la atención tutorial orientadora, personalizada y aplicada a contextos específicos cobra un papel fundamental. No hay que desdeñar la clase magistral, siempre que esté orientada a la consecución de las competencias. De igual modo hay que facilitar los materiales y recursos que faciliten el aprendizaje autónomo. La planificación puede materializarse a través de distintos documentos (plan docente, guías docentes...) y difundirse por distintas vías: en una plataforma, en la web de la asignatura, en un tablón, en un documento en papel...

En cuarto lugar, otra implicación importante, es el trabajo colaborativo del profesorado. Debemos ser conscientes de que la adquisición de los objetivos competenciales escapa del ámbito de la asignatura; nos atreveríamos a decir que, al menos para algunas de ellas, incluso escapa del ámbito del curso y deben ser considerados más bien como objetivos de ciclo o titulación. Por ello deben ser trabajadas conjuntamente, con una distribución a lo largo de todo el desarrollo curricular previamente programada por los profesores y, en consecuencia, el grado de adquisición de las mismas debe ser evaluado colectivamente. Aspecto, este último, muy importante dado el grado de subjetividad que pude tener alguna de estas valoraciones y que, al ser evaluado colectivamente e incluso por pares, se verá equilibrado por la opinión de los distintos implicados.

En quinto lugar, el reto de la evaluación de las competencias. Este modelo afecta también la evaluación al trasladarse del suministro a los resultados (from input to output) y a los procesos y contextos del que aprende. Se hace necesario ampliar el concepto de evaluación del rendimiento para que abarque los diferentes componentes de las competencias personales y profesionales que se propone desarrollar la enseñanza universitaria: conocimientos, habilidades, actitudes y comportamientos.

Por último, el lugar de las prácticas. El profesor debe abrir el campo de la docencia y del aprendizaje al mundo real del ejercicio de las profesiones concretas. Las prácticas y las demandas de estas en términos de competencias profesionales deben vertebrar el diseño curricular de la titulación, teniendo en cuenta esta doble coordenada de competencias generales y específicas o técnico profesionales y de las dimensiones de saber, saber hacer y transferir para aprender a desaprender (la interrelación entre el conocimiento, la praxis de la profesión y la fundamentación en la formación académica)

\section{Referencias}

Bain, K. (2005). Lo que hacen los mejores profesores de universidad. Traducido por

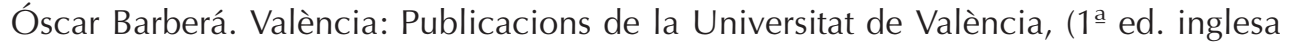
2004).

Biggs, J. (2006). Calidad del aprendizaje universitario. Madrid: Narcea.

Boreham, N. (2004). A Theory of collective competence: Challenging the neo-liberal individualisation of performance at work. British Journal of Educational Studies, 52, 1, 5-17.

Blanco, A. (2007). Trabajadores competentes. Madrid: Esic.

Bricall et al. (2000). Informe 2000. Madrid: CRUE.

Bunk, G. P. (1994). La transmisión de las competencias en la formación y perfeccionamiento de profesionales en la RFA. Cedefop. 
Corominas, E.; Tesouro, M.; Capell, D.; Teixidó, J.; Pèlach, J. y Cortada, R. (2006). Percepciones del profesorado ante la incorporación de las competencias genéricas en la formación universitaria. Revista de Educación, 341. Septiembre-diciembre, 301-336.

De la Cruz, Ma A. (2003). Taller sobre el proceso de aprendizaje-enseñanza de competencias. Zaragoza: Instituto de Ciencias de la Educación. Universidad de Zaragoza.

De Miguel, M. (2003). Calidad de la enseñanza universitaria y desarrollo profesional del profesorado. Revista de Educación, 331, 13-34.

Domènech, J. y Viñas, J. (1997). La organización del espacio y del tiempo en el centro educativo. Barcelona: Graó.

Freire, P. (1998). Pedagogía de la autonomía. Madrid: Siglo XXI.

Fullan, M. (1988). "Research into educational innovation". En R. LATTER et al. Understanding school managemen.t London: Open University Press.

Fullan, M. (1994). "Coordinating top-down and bottom-up strategies for educational reform". En R.J. ANSON (comp.). Systemic Reform: Perspectives on Personalizing Education. Washington, DC: US Department of Education Report.

Fullan, M. (2002). Liderar en una cultura de cambio. Barcelona: Octaedro.

Goble, N. y Porter, J. F. (1980). La cambiante función del profesor. Madrid: Narcea.

González, J. y Wagenaar, R. (2003). Tuning Educational Structures in Europe. Bilbao: Universidad de Deusto. Proyecto Tuning. http://www.relint.deusto.es/TUNINGProject/index.htm.

Hannan, A. (2006). Innovación en la enseñanza superior, la. Enseñanza, aprendizaje y culturas institucionales. Madrid: Narcea.

Ibarra, A. (2000). Formación de Recursos Humanos y Competencia Laboral. Montevideo: Boletín Cinterfor/OIT No. 149.

Jarvis, P. (2006). Universidades corporativas. Nuevos modelos de aprendizaje en la Sociedad Global. Madrid: Narcea.

Lasnier, R. (2000). Réussir la formation par compétences. Montreal: Guérin.

Le Boterf, G. (2001). Ingeniería de las competencias. Barcelona: Gestión 2000.

Lesourne, J. (1993). Educación y sociedad. Desafíos para el año 2000. Barcelona: Oikos- Tau.

Lévy-Levoyer, C. (1997). Gestión de competencias. Barcelona: Gestión 2000.

Martín, M. (1989): "Mitos y creencias". Comunicación Social. Tendencias. Madrid: Fundesco, 193-203.

McClelland, D. and Spencer, S.M. (1994). Competency Assessment Methods. History and State of the Art. Hay/Mc: Research Press. MERTENS, L. (1996). Competencia laboral: sistemas, surgimiento y modelos. Montevideo: OIT/CINTERFOR.

MEC (2006). Borrador de propuesta Directrices para la Elaboración de Titulos Universitarios de Grado y Máster. Madrid: MEC. 
MEC (2006). Propuestas para la renovación de las metodologías en la universidad. Madrid: Secretaría de Estado de Universidades e Investigación. Secretaría General Técnica.

Monereo, C. y Pozo, J. I. (Eds) (2003). La universidad ante la nueva cultura educativa. Madrid: Síntesis.

Mora, J. G. (2003). Competencias y empleo de los jóvenes graduados universitarios. Revista de Educación, 330, 157-170.

Prieto, J. M. (1997). Prólogo. En C. LÉVY-LEVOYER. Gestión de las competencias. Barcelona: Gestión 2000.

Quintanilla, M. A. (1998). "El reto de la calidad en las universidades". En J. PORTA Y M. LLANODOSA (coords.). La universidad en el cambio de siglo. Madrid: Alianza.

REAL ACADEMIA ESPAÑOLA (1995). Diccionario de la Lengua Española. Madrid: Espasa Calpe, DL.

Rodríguez, Mำ L. (2006). Evaluación, balance y formación de competencias laborales transversales. Barcelona: Laertes.

Rué, J. (2007). Enseñar en la Universidad. El EEE como reto para la educación superior. Madrid: Narcea.

Shön, D. A. (1983). The reflective practitioner. How professionals think in action. London: Temple Smith.

Shön, D. A. (1987). Educating the reflective practitioner. London: Jossey Bass Publishers.

Tedesco, J. C. (1997). El Nuevo pacto educativo. Educación competitividad y ciudadanía en la sociedad moderna. Madrid: Anaya.

Tejada, J. (1999). Acerca de las competencias Profesionales. Revista Herramientas, 22, 47-58.

Villa, A. (1997). "Formación del profesorado en la investigación orientada al cambio". En VVAA. Atreverse a Educar. Madrid: Narcea.

Yániz, C. y Villardón, L. (2006). Planificar desde competencias para promover el aprendizaje. Bilbao: Mensajero.

Zabalza, M. A. (2007). Enseñanza universitaria. El escenario y sus protagonistas. Madrid: Narcea.

Zarifian, P. (2001). Le modèle de la compétence. Paris: Éditions Liaisons. 